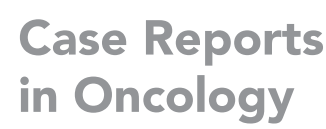

Case Reports

in Oncology

\title{
A Rare Case of Neglected Rupture of Right Axillary Artery Pseudoaneurysm Mimicking a Soft Tissue Tumor
}

\author{
Rosy Setiawati ${ }^{a}$ Vivid Umi Varidha ${ }^{a}$ Giuseppe Guglielmi ${ }^{\text {b c }}$ \\ Filippo Del Grande ${ }^{d}$ \\ aDepartment of Radiology, Faculty of Medicine, Airlangga University, Surabaya, Indonesia; \\ bepartment of Clinical and Experimental Medicine, School of Medicine, Foggia University, \\ Foggia, Italy; 'Department of Radiology, School of Medicine, Foggia University, Foggia, \\ Italy; ${ }^{d}$ Department of Radiology, Ente Ospedaliero Cantonale, Bellinzona, Switzerland
}

\section{Keywords}

Rare case $\cdot$ Pseudoaneurysm of the axillary artery $\cdot$ Neglected pseudoaneurysm mimicking soft tissue tumor

\begin{abstract}
Traumatic lesions of the axillary artery itself are limited to $2.9-9 \%$ of major arterial injuries. Pseudoaneurysms represent a pulsating encapsulated hematoma in communication with the lumen of a ruptured vessel. Traumatic pseudoaneurysm of the axillary artery is a rare sequela of injury to the shoulder region. We describe a case of posttraumatic pseudoaneurysm involving the axillary artery, which was initially misdiagnosed as an aggressive soft tissue tumor. The man presented 10 years after an injury from a fall from a tree with a slowly growing mass in the right upper limb region and reduced range of movement. This is a neglected case with a history of traditional massage. The patient presented a pathologic fracture of the right proximal humerus and dislocation of the glenohumeral joint. At the beginning, it was suggested to be a primary soft tissue tumor, but after several examinations, including comparable X-ray, ultrasound, and histopathology, the results did not support a soft tissue tumor. Magnetic resonance imaging and computed tomography angiography (CTA) finally confirmed the finding of a pseudoaneurysm of the right axillary artery associated with a huge hematoma with different age of the bleeding product and granulation tissue. This case demonstrates the necessity of early diagnosis of axillary artery pseudoaneurysm to prevent complications after a history of trauma. CTA is a useful modality to evaluate vascular injury and provides valuable information.




\section{Introduction}

Injury to the axillary artery after trauma of the shoulder is extremely rare. Traumatic lesions of the axillary artery itself are limited to $2.9-9 \%$ of major arterial injuries [1]. Injury to the shoulder region is the most common complication of a dislocated shoulder joint, humeral fractures, plexus brachialis injuries, and also vascular injury [1-15]. Pseudoaneurysms represent an encapsulated hematoma in communication with the lumen of a ruptured vessel [1-17]. Traumatic pseudoaneurysm of the axillary artery is a rare sequela of injury to the shoulder region $[1,3,5,9,13]$.

\section{Case Report}

We present a rare case of a 67-year-old male patient admitted to our hospital with a slowly growing mass in the right upper extremity. The patient reported that he fell from a tree 10 years prior to the hospital admission. After a year of injury, the patient had begun to complain of a lump with a gradually enlarging mass in the axillary region. No history of neurological deficit was found. Our physical exam showed a firm, tender, nonpulsatile and immobile mass with unclear boundary and we suspected a primary soft tissue tumor (Fig. 1).

$\mathrm{X}$-ray examination showed destruction of the right proximal humerus with glenohumeral dislocation and a large soft tissue mass surrounding it. An oval-shaped eggshell calcification was also found in the axillary region. Marked erosion was seen at the adjacent glenoid fossa and acromion with sclerotic changes with widening of the glenohumeral joint space. Based on the imaging findings, we suspected a malignant soft tissue tumor with following bone destruction (Fig. 2).

To better investigate the suspected soft tissue mass, we performed an ultrasound exam followed by an ultrasound-guided fine needle aspiration biopsy. A large soft tissue mass of the right shoulder with multiple cystic hypoechoic foci and deformities of the surrounding bone was found. These findings led to a feature of primary soft tissue tumor (Fig. 3). No significant abnormal vascular filling was found on color Doppler ultrasound; the mass size was too large with limited ultrasound frequency penetration.

Regarding the indication of a soft tissue tumor, ultrasound-guided fine needle aspiration biopsy was obtained, leading to the conclusion of a chronic suppurative inflammation and no
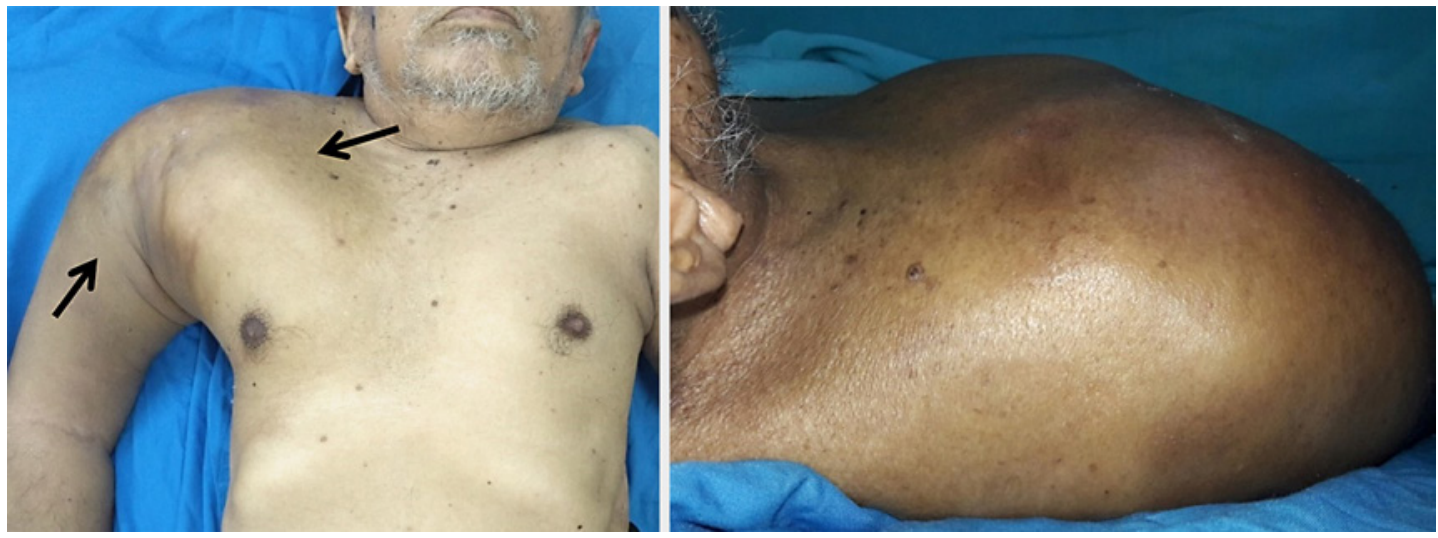

Fig. 1. Clinical photograph of the pseudoaneurysm of the right axillary artery. A large growing mass of the right shoulder showed firm palpation, warm, unclear boundary, and tenderness; it was immobile and nonpulsatile (black arrows). 


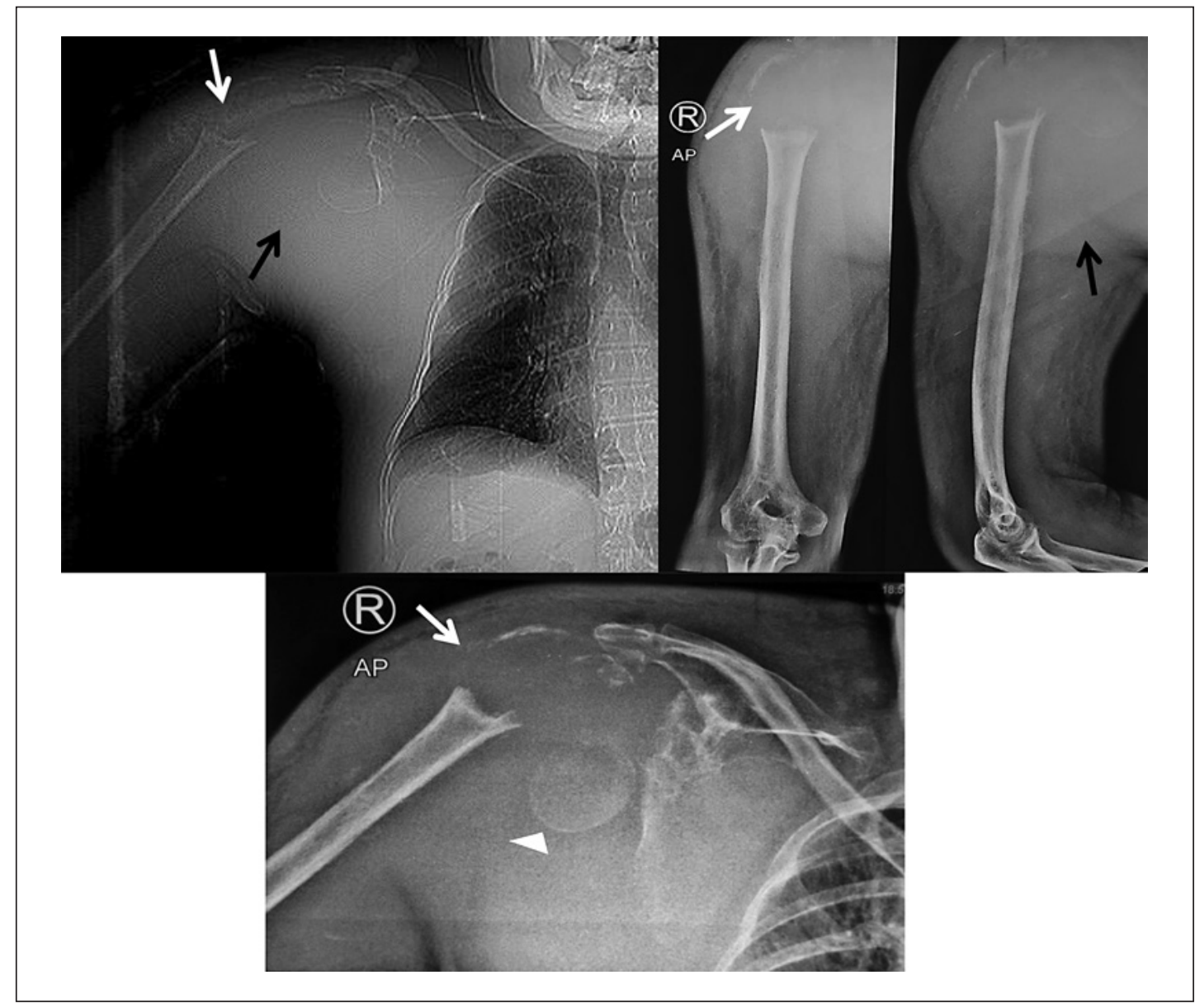

Fig. 2. Right anterior posterior (AP) shoulder and AP lateral humerus radiographs. Radiograph showed destruction of the right proximal humerus with superolateral glenohumeral joint dislocation with a large soft tissue swelling surrounding it (white arrows). Marked erosion was seen at the adjacent glenoid. An ovalshaped eggshell calcification was also found in the axillary region (white arrowhead).

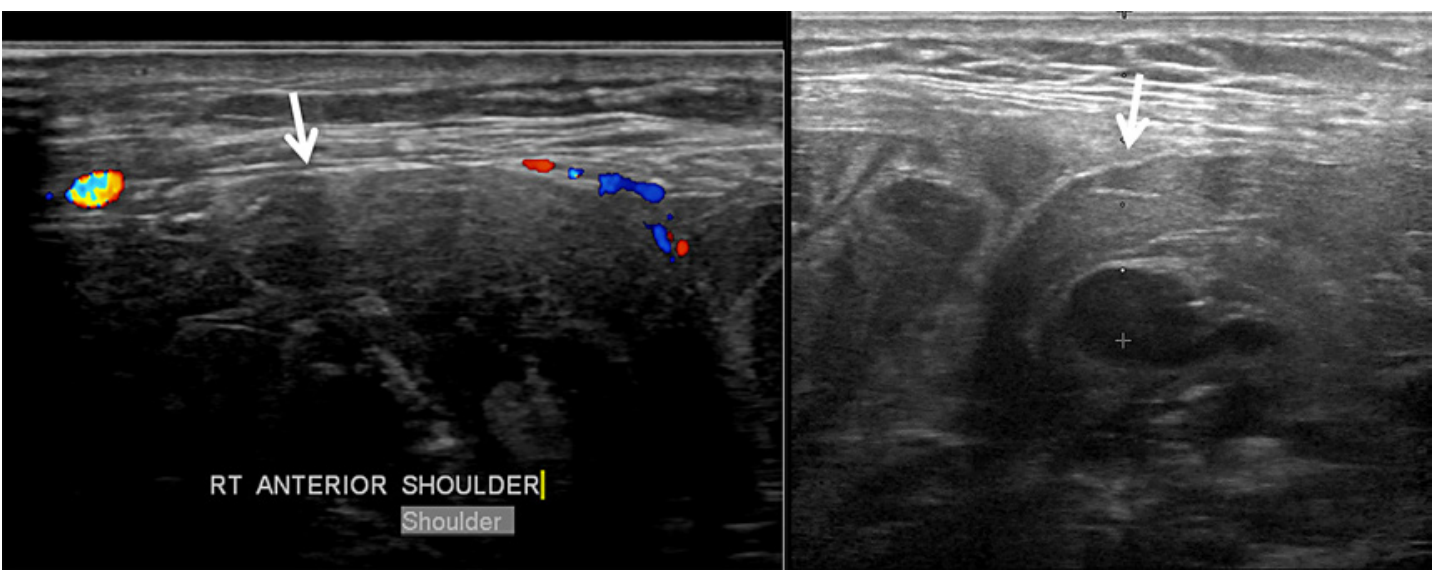

Fig. 3. Ultrasonography of the right shoulder. A large soft tissue mass of the right shoulder was seen with multiple cystic hypoechoic foci which were lacking pulsation on color Doppler. 


\section{Case Reports in Oncology}
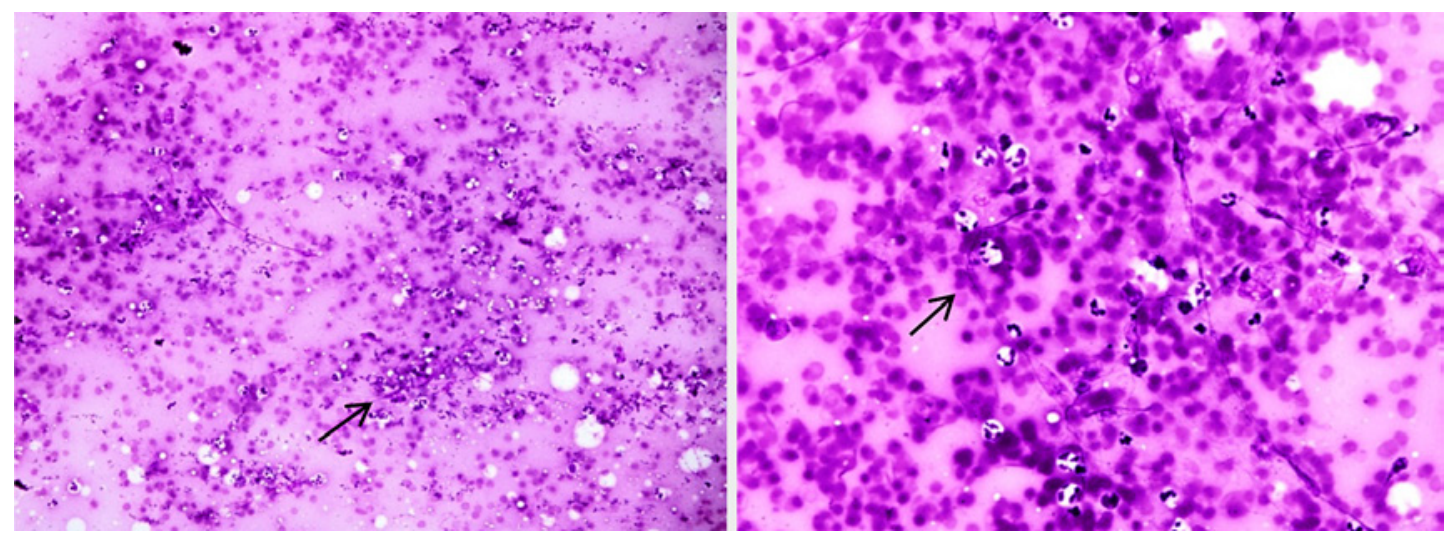

Fig. 4. Histopathology results showed a chronic suppurative inflammation and no sign of malignancy.

visible signs of malignancy (Fig. 4). The needle biopsy result was inconclusive, and further imaging investigations were performed.

1.5-T magnetic resonance imaging (MRI) exam confirmed a large mixed cystic semisolid soft tissue mass of the right shoulder with bone destruction of the right proximal humerus as well as glenohumeral joint dislocation. Hemorrhagic foci were found as hyperintense signals on T1-weighted image (T1WI), and a hypointense rim was found in the peritumoral region on T2-weighted image (T2WI). In the axilla region, there was also an oval lesion with multilayer walls which consisted of mixed hypointense and hyperintense signals suggesting a vascular aneurysm with different age of the bleeding product. After contrast administration, this oval lesion revealed relatively homogenous contrast filling located in the central part of the massive mass in the right axillary region and rim contrast enhancement in the surrounding muscle structures seen on coronal and axial T1WI, T2WI, and T1WI with contrast sequences (Fig. 5). We suspected a neglected axillary artery pseudoaneurysm due to previous humeral head trauma.

Computed tomography (CT) scan was performed to confirm the finding of a pseudoaneurysm and demonstrated the presence of a rounded contrast-enhanced lesion indicating a right saccular axillary artery pseudoaneurysm with a narrow neck approximately $5.1 \mathrm{~mm}$ in diameter and with a height of $3.5 \mathrm{~cm}$ as well as a width of $3.2 \mathrm{~cm}$ projecting laterally to the axillary artery (Fig. 6).

Surgical exploration was planned. Unfortunately, this procedure could not be performed because of the unstable condition of the patient due to a sepsis complication with elevation of C-reactive protein of $11.10 \mathrm{mg} / \mathrm{dL}$ and a decreased $\mathrm{Hb}$ level. Finally, this patient passed away because of sepsis.

\section{Discussion}

Pseudoaneurysms of the axillary artery are a rare complication after blunt trauma in the axillary region [1-15]. In the case of blunt trauma, a shoulder joint-related bone injury may occur, and most often an anterior dislocation [3-5, 8]; axillary artery damage has been reported with a prevalence of about $0.3 \%[2,3]$. Other injuries that can occur are fractures of the humeral neck and the proximal humerus [5]. In the reports by Erler et al. [16] and Kim et al. [17], the diagnosis of pseudoaneurysms was not straightforward. This pathologic condition often does not occur shortly after trauma, but it develops later [5]. Indeed, clinical suspicion 


\section{Case Reports in Oncology}

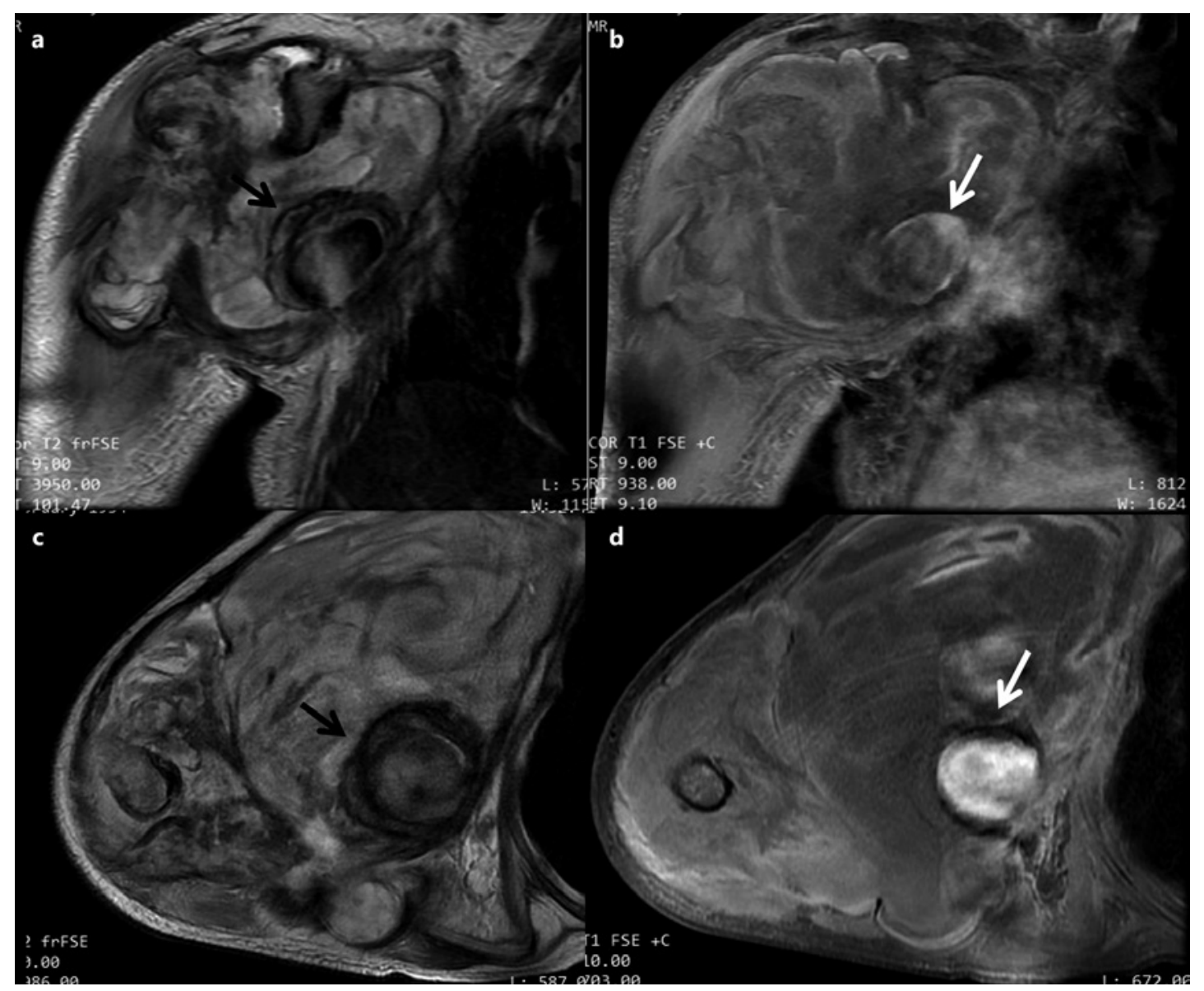

Fig. 5. Shoulder MRI on axial-coronal T2WI (a, c) and T1 fat-saturated gadolinium-based contrast (b, d). MRI showed a mixed cystic semisolid mass involving the surrounding muscles in the right shoulder. The mass consisted of an oval lesion in the axillary region with multilayer thick walls of mixed hypointense and hyperintense signal intensities. After contrast administration, it showed a relatively homogenous oval-shaped contrast filling (white arrow) located in the central part of the massive mass in the right axillary region, confirming a right axillary artery pseudoaneurysm with different age of the bleeding product and rim contrast enhancement in the granulation tissues.

can be lessened by the absence of hard initial signs of arterial injury. It is very important to raise suspicion of a pseudoaneurysm in order to avoid complications such as brachial plexus injury [5]. Since this patient presented late, without any typical features of pseudoaneurysm, it was misdiagnosed as a soft tissue tumor. We reported a rare case of a patient with a missed ruptured axillary artery pseudoaneurysm who underwent several radiological examinations and fine needle aspiration biopsy.

An aneurysm is a bulging, weakened area in the wall of a blood vessel resulting in an abnormal widening or ballooning greater than $50 \%$ of the vessel's normal diameter. An aneurysm may occur in any blood vessel, but is more often seen in an artery rather than a vein [1]. Aneurysms can develop in all arteries of the human body. Morphologically, there are 2 main types of aneurysms: saccular and fusiform aneurysms. On the other hand, pseudoaneurysm, or false aneurysm, is not an enlargement of any of the layers of the blood vessel wall. A false aneurysm may be the result of a prior surgery or trauma. Sometimes, a tear can occur on the inside layer of the vessel. As a result, blood fills in-between the layers of the blood 

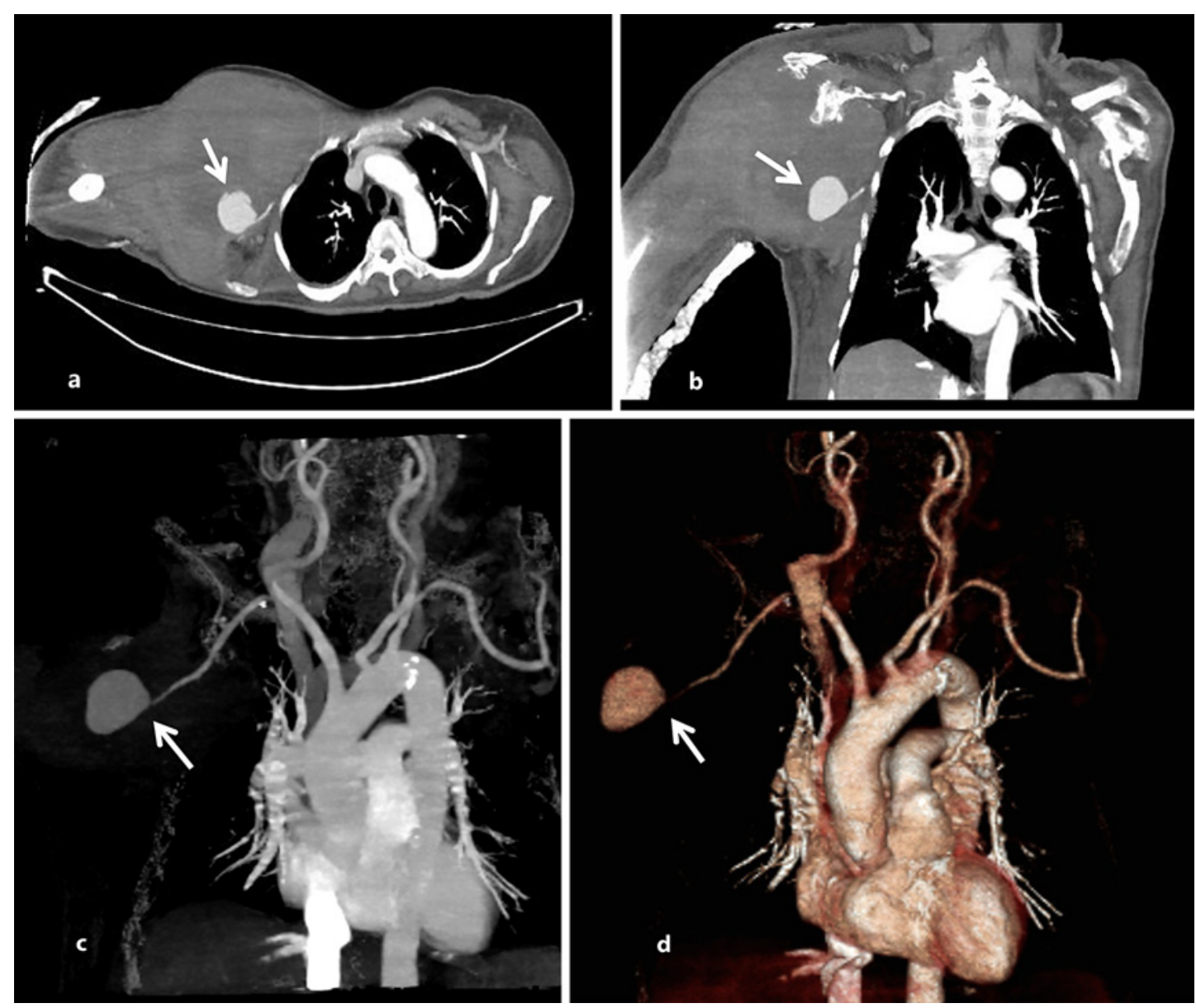

Fig. 6. CTA of the right shoulder in axial-coronal slices (a, b), coronal MIP (Maximum Intensity Projection) (c), and volume-rendered (d) images. CTA showed an oval contrast-enhanced lesion indicating a right saccular axillary artery pseudoaneurysm with a narrow neck approximately $5.1 \mathrm{~mm}$ in diameter and a height of $3.5 \mathrm{~cm}$ as well as a width of $3.2 \mathrm{~cm}$ projecting laterally to the normal axillary artery.

vessel wall creating a pseudoaneurysm [10]. The pathogenesis of axillary artery pseudoaneurysm is still debatable. The anatomical position of the vessel is thought to play a role in its susceptibility to trauma. Some studies have suggested that injury may arrive as a consequence of the position of the axillary artery, fixed between the anterior and posterior circumflex arteries and the scapular arteries. Another theory sustains that movement of the humeral head into hyperabduction compresses and distorts the vessel against the pectoralis minor; moreover, the axillary artery displays a degree of anatomical variation in its course, which may contribute further to this susceptibility to injury [11].

The complications of pseudoaneurysm can be rupture, distal thromboembolism, and the effects of compression. Infection, polyarteritis nodosa, congenital arterial defects, and especially trauma play a role in the pathogenesis of upper extremity pseudoaneurysms. If the only causal factor is trauma, the aneurysm takes the form of a pseudoaneurysm [3]. The early diagnosis of upper-limb pseudoaneurysms should prevent the risk of vascular and neurological compromises with potential serious long-term sequelae [11]. When a pseudoaneurysm is clinically suspected, imaging modalities, such as ultrasound examination, CT angiography (CTA), magnetic resonance angiography, or digital subtraction angiography (DSA), may be 
useful to assist in the diagnosis [1]. Compared to other imaging modalities, ultrasonic examination is cheap and noninvasive and has the advantage of being able to be performed at the patient's bedside. DSA, employed as the definitive diagnostic modality for vascular injury, has the advantage of enabling the placement of a stent or occluding balloon as an endovascular treatment as required. However, conventional diagnostic DSA also has the disadvantage of being time consuming and invasive, and may be associated with serious complications. CTA is a modality to study arterial anatomy, with the additional advantages of being noninvasive and able to evaluate arterial trauma rapidly and reliably, reducing the delay before repair of the injury $[1,3,7]$. Misdiagnosis or delayed diagnosis of axillary pseudoaneurysm may result in upper limb morbidity or patient mortality [8].

In the presented case, diagnosis of pseudoaneurysm was delayed because the clinical presentation was obscured by a soft tissue mass suspected to be a primary soft tissue tumor. Initial radiological and histopathologic examinations led to a primary soft tissue mass. Following further evaluation using MRI resulted in the finding of a right axillary artery pseudoaneurysm with a huge hematoma involving the muscle structures with different age of the bleeding product. Finally, the diagnosis was confirmed by CTA examination. The patient suffered from an infection complication causing the mortality.

Axillary artery pseudoaneurysms are rarely found in axillary region trauma [1-5]; in this case, the presence of axillary artery pseudoaneurysm may be due to trauma and may be aggravated by traditional massage. As described by some authors, pseudoaneurysm may occur immediately after primary or delayed injury, due to an initially small aneurysm size and a good collateral on the upper extremities [1]. Such cases have also been reported by several authors, including Dympep et al. [3], who reported some cases in patients with left shoulder region trauma with axillary artery pseudoaneurysms mimicking soft tissue tumors with brachial plexus injury. The diagnosis was done with Doppler ultrasound, which resulted in confirmation by CTA [3]. Therefore, a good communication between clinician and radiologist is needed when handling any case by exploring the patient's disease history, so that an optimal imaging diagnosis can be reached immediately and then optimal therapy can be done to avoid the worst complication that may cause the patient's mortality.

\section{Conclusion}

Doppler ultrasound, DSA, CTA, and magnetic resonance angiography are useful modalities when evaluating arterial injury. CTA is a modality to study arterial anatomy, with the additional advantages of being noninvasive and able to evaluate arterial trauma rapidly and reliably, reducing the delay before repair of the injury; however, delayed diagnosis of axillary pseudoaneurysm may result in upper limb morbidity or patient mortality.

\section{Acknowledgment}

Special thanks to Zahrona Kusuma for completing the data.

\section{Statement of Ethics}

Ethical approval for this study was obtained from Dr. Soetomo General Hospital ethics committee (400/03/K.3/CR/302/2018). The authors confirm that written informed consent was obtained from the patient for the publication of the case (including publication of images).

\section{Karger'}




\section{Conflict of Interest Statement}

The authors have no conflicts of interest to declare.

\section{Funding Sources}

We did not receive any funding.

\section{Author Contributions}

Rosy Setiawati contributed in preparing the case report design, collecting and analyzing the data of the patient, and writing the manuscript. Vivid Umu Varidha contributed in collecting data of the patient and writing the manuscript. Giuseppe Guglielmi contributed in reviewing this article and advising on methodology design. Filippo Del Grande contributed in reviewing this article, advising on methodology design, and analyzing data.

\section{Data Availability}

1. The case report data used to support the findings of this study are included in the article.

2. The case report data are not under current embargo while the case findings are commercialized.

3. The case report data used to support the findings of this study may be released upon application to Airlangga University by contacting the email address rosy-s@fk.unair. ac.id.

\section{References}

1 Chen L, Peng F, Wang T, Chen D, Yang J. Traumatic pseudoaneurysm of axillary artery combined with brachial plexus injury. PLoS One. 2014;9(11):e113099.

2 Moss AJ, Valenti D, Fraser SC, Murie J. An unusual shoulder injury. J Surg Case Rep. 2011;2011(10):9.

3 Dympep B, Khangarot S, Hadke N. An unusual presentation of traumatic pseudoaneurysm of axillary artery mimicking soft tissue tumor. J Surg Case Rep. 2012;2012(10):17.

4 Bertrand JC, Maestro M, Péquignot JP, Mouiel J. [Vascular complications of simple anterior dislocations of the shoulder. Three cases]. Ann Chir. 1982;36(5):329.

5 Fitzgerald JF, Keates J. False aneurysm as a late complication of anterior dislocation of the shoulder. Ann Surg. 1975;181(6):785.

6 Gallen J, Wiss DA, Cantelmo N, Menzoin JO. Traumatic pseudoaneurysm of the axillary artery: report of three cases and literature review. J Trauma. 1984;24(4):350-2.

7 Goncu T, Toktas F, Tiryakioglu O, Yumun G, Demirtas S, Yavuz S. Posttraumatic true aneurysm of the axillary artery following blunt trauma. Case Rep Med. 2010;210391.

8 Whittam K, Hardy M. A case study of an axillary artery pseudoaneurysm following anterior dislocation of the glenohumeral joint: a rare presentation on plain film radiographs. Radiography. 2007;13(3):221-8.

9 Palcau L, Gouicem D, Dufranc J. Delayed axillary artery pseudoaneurysm as an isolated consequence to anterior dislocation of the shoulder. Ann Vasc Surg. 2012;26(2):279.e9-279.e12.

10 Mehrpooya M, Salehi M, Eskandari R, Shajirat Z, Golabchi A, Mazoochi M. Diagnostic dilemma: saccular aneurysm or pseudoaneurysm of the ascending aorta with dissection above level of leaflets. ARYA Atheroscler. 2012;8(3):167-9.

11 Songur M, Sahin E, Kalem M, Zehir S. Amputation for upper extremity ischemia following shoulder dislocation: case report and a review of literature. Eur Res J. 2015;1(2):66.

12 Schumann DR, Superti MJ, Seyboth FC, Jacomel GE. Brachial plexus injury secondary to pseudoaneurysm of axillary artery after glenohumeral dislocation: case report. Rev Bras Ortop. 2017;52(4):491-5.

\section{Karger's}


13 Sandiford N, Tsitskaris K, Erritty M. Delayed presentation of a pseudoaneurysm of the subclavian and axillary artery - the importance of vigilance. J R Soc Med. 2010;103(2):67-9.

14 Akcali Y, Kahraman C, Ozcan N, Kontas O. A giant aneurysm of a branch of axillary artery. J Vasc Surg. 2008; 47(4):881.

15 Gasparini M, Jakomin T. Endovascular treatment of an axillary artery pseudoaneurysm after anterior shoulder dislocation: a case report. Zdrav Vestn. 2011;80(5):422-6.

16 Erler K, Ozdemir MT, Oguz E, Basbozkurt M. Does false aneurysm behave like a sarcoma? Distal femoral arterial false aneurysm simulated a malign mesenchymal tumor. A case report and review of the literature. Arch Orthop Trauma Surg. 2004;124(1):60-3.

17 Kim YJ, Baek WK, Kim JY, Park SW, Jeon YS, Lee KH, et al. Pseudoaneurysm of the popliteal artery mimicking tumorous condition. J Korean Surg Soc. 2011;80(Suppl 1):S71-74. 